[0212-7199 (2007) 24: 5; pp 212-216] ANALES DE MEDICINA INTERNA Copyright (C) 2007 ARAN EDICIONES, S.L.

AN. MED. INTERNA (Madrid) Vol. 24, N. ${ }^{\circ}$ 5, pp. 212-216, 2007

\title{
Evolución de la ansiedad y de la depresión detectada durante la hospitalización en un servicio de Medicina Interna
}

\author{
A. GRAU, P. COMAS, R. SUÑER ${ }^{1}$, E. PELÁEZ ${ }^{2}$, L. SALA², M. PLANAS ${ }^{2}$ \\ Servicio de Medicina Interna. Hospital de Figueres. ${ }^{1}$ Universidad de Girona. ${ }^{2}$ Unidad \\ Docente de Medicina Familiar y Comunitaria. Girona
}

EVOLUTION OF ANXIETY AND DEPRESSION DETECTED DURING HOSPITALIZATION IN AN INTERNAL MEDICINE SERVICE

\begin{abstract}
RESUMEN
Objetivo: Estudiar la evolución de los síntomas de ansiedad (As) y depresión (Dp) a los dos meses del alta hospitalaria y los factores asociados con su persistencia.

Método: Estudio longitudinal observacional. Los pacientes con As o Dp, detectada mediante el cuestionario Hospital Ansiedad y Depresión (HAD) en el cuarto día de estancia hospitalaria, fueron seguidos durante dos meses sin recibir psicofármacos. Se valoraron factores sociodemográficos, historia psiquiátrica, estado funcional, comorbilidad y el HAD del estado que tenía tres semanas antes del ingreso.

Resultados: Se siguieron 38 pacientes con As (22 varones), edad media 62,2 años, y 35 pacientes con Dp (22 varones), edad media 68,1 años. Persistieron con síntomas de As a los dos meses 23 pacientes (60,5\%; IC95\%: 43,4 - 76) y con Dp 18 (51,4\%; IC95\%: 34 - 68,6). La As previa al ingreso y un menor nivel de estudios se asociaron con persistencia de As, y la edad de 70 o más años, sexo femenino, estudios primarios, Barthel durante el ingreso menor a 100 y depresión en las tres semanas previas se asociaron con persistencia de Dp.

Conclusiones: Persiste la sintomatología en más de la mitad de los pacientes. La valoración del HAD previo al ingreso puede ayudar a decidir la prescripción de tratamiento.
\end{abstract}

PALABRAS CLAVE: Ansiedad. Depresión. Pacientes hospitalizados. Pronóstico.

\begin{abstract}
Objective: To study the evolution of the symptoms of anxiety (Ax) and depression $(\mathrm{Dp})$ two months after hospital release and factors associated with their persistence.

Method: An observational longitudinal study. Patients with Ax or $D p$, evaluated by the Hospital Anxiety and Depression (HAD) scale on the fourth day of hospitalisation, were followed over two months without the use of psychopharmacological drugs. Sociodemographic factors, psychiatric history, functional state, comorbidity and the HAD stage three weeks before admission were evaluated.

Results: Thirty-eight patients with Ax (22 males) with a mean age of 62.2 years and 35 patients with $\mathrm{Dp}(22$ males) with a mean age of 68.1 years were studied. Symptoms of Ax persisted in 23 patients $(60.5 \%$; C195\%: 43.4-76) and symptoms of Dp persisted in 18 (51.4\%; CI95\%: 34-68.6). Ax before admission and a lower educational level were associated with the persistence of Ax. An age of 70 or more, female gender and primary studies, Barthel Index on admission of < 100 and depression in the three weeks before admission were associated with persistence of $\mathrm{Dp}$.

Conclusions: Symptoms persisted in over half of the patients. The evaluation of HAD before admission may help in determining the treatment course to be followed.
\end{abstract}

KEY WORDS: Anxiety. Depression. Inpatients. Prognosis.

Grau A, Comas P, Suñer R, Peláez E, Sala L, Planas M. Evolución de la ansiedad y depresión detectada durante la hospitalización en un servicio de Medicina Interna. An Med Interna (Madrid) 2007; 24: 212-216.

\section{INTRODUCCIÓN}

La ansiedad (As) y la Depresión (Dp) son trastornos psiquiátricos de alta prevalencia entre los pacientes ingresados por causas orgánicas en salas médicas o quirúrgicas de hospitales generales. Utilizando los criterios diagnósticos del DSM recogidos mediante entrevista estructurada las cifras de prevalencia oscilan entre el 7 al 20\% para Dp y del 6 al 17\% para As (1-3). Cuando la valoración se realiza atendiendo a la presencia de síntomas recopilados mediante cuestionarios valida- dos las cifras de prevalencia aumentan y se sitúan entre el 16 y el $43 \%$ para la Dp $(1,4-8)$, y entre el 16 y el $30 \%$ para la As $(2,6-9)$.

En una muestra representativa de la población española no institucionalizada formada por 5.473 individuos mayores de 18 años, la prevalencia de trastornos del estado de ánimo (Dp) en algún momento de la vida fue de una $6,7 \%$ para los varones y de un $15,9 \%$ para las mujeres, y la prevalencia de trastornos de As durante la vida se estimó en 5,7\% para los varones y $12,8 \%$ para las mujeres (10). 
En los pacientes ingresados es frecuente que no se prescriba tratamiento cuando se detectan síntomas de As o Dp $(2,11,12)$ porque es atribuida al proceso de la hospitalización y a la enfermedad orgánica asociada. Un estudio evolutivo de los síntomas de As y Dp durante el ingreso en el hospital observó un descenso de los síntomas en los tres primeros días de estancia hospitalaria con una posterior estabilidad (13). Se han realizado pocos estudios que contemplen un seguimiento posterior al alta.

Ante la pregunta de si se debe tratar a los pacientes con sintomatología de As o Dp detectada durante la hospitalización, o si es más correcto esperar a una nueva evaluación ambulatoria, diseñamos esta investigación con el objetivo de conocer la evolución natural de los síntomas de As y Dp a los dos meses del alta hospitalaria y los factores asociados a la persistencia de los síntomas psiquiátricos.

\section{SUJETOS Y MÉTODOS}

Se diseñó un estudio longitudinal, prospectivo y observacional. Se realizó un muestreo consecutivo que incluyó a 918 pacientes con un ingreso superior a cuatro días en la sala de medicina interna del Hospital de Figueres desde agosto del 2004 hasta marzo del 2005. Se excluyeron del estudio a 456 pacientes. Presentaban algún criterio de exclusión 346 pacientes al estar diagnosticados de demencia, hallarse bajo tratamiento antidepresivo o ansiolítico, tener un estado físico o mental que no les permitía contestar adecuadamente los cuestionarios o por dificultad idiomática. También se excluyeron a 44 pacientes que no aceptaron participar en el estudio. A todos los pacientes con edad superior a los 65 años se les valoró el estado cognitivo mediante el test de Pfeiffer $(14,15)$ y fueron excluidos 66 pacientes porque cometieron más de tres errores en dicho test (Fig. 1). Todos los participantes fueron informados del objetivo y de la metodología del estudio y firmaron el consentimiento. El protocolo fue aprobado por el Comité de Ética en Investigación Clínica del Hospital Josep Trueta de Girona.

Los participantes del estudio respondieron el cuestionario Hospital Ansiedad Depresión (HAD) (16-20). El HAD es un cuestionario con 7 afirmaciones para valorar As y 7 para Dp, con puntuaciones que oscilan de 0 a 21 para cada subescala. Es una medida de estado que explora la situación en la última semana. Ideado para ser administrado a sujetos con enfermedades orgánicas se han eliminado los aspectos físicos que pueden acompañar a la As o a la Dp incidiendo sólo en los emocionales. Además de la valoración cuantitativa puede estratificarse en no As o no Dp si los valores de HAD son inferiores a 8 , posible o dudosa entre 8 y 10 , y probable o afirmativa si son de 11 o superiores.

El cuarto día de estancia hospitalaria se les solicitó que cumplimentaran el HAD actual, que correspondía a su situación durante el ingreso. También se les solicitó que realizaran el esfuerzo de contestar el cuestionario HAD rememorando qué síntomas percibían 3 semanas antes del ingreso, para obtener el denominado HAD previo, que valora el estado precedente al ingreso en el hospital.

Los participantes con un HAD actual de 8 o superior en una de las subescalas fueron incluidos en el grupo correspondiente de As o de Dp. Se registraron variables sociodemográficas, historia de As y Dp, medicación previa para As o Dp, el índice de comorbilidad de Charlson (21) y la escala de Barthel (22). A los participantes no se les prescribió psicofármacos ni durante el ingreso ni al alta. Se les citó para volver a valorar la presencia de síntomas de As o Dp mediante el HAD a los dos meses del alta hospitalaria.

Análisis estadístico: Se calculó el porcentaje de pacientes que permanecían con síntomas de As o Dp a los dos meses. Se compararon las valoraciones cuantitativas de cada escala del HAD en los diferentes tiempos de medida, mediante la $\mathrm{T}$ de Student para datos pareados. Se calculó el riesgo relativo de permanecer con síntomas en relación a la presencia de variables cualitativas.

\section{RESULTADOS}

De los 918 pacientes inicialmente evaluados se administró el cuestionario HAD a 462 pacientes después de aplicar los criterios de exclusión, obtener el consentimiento y detectar

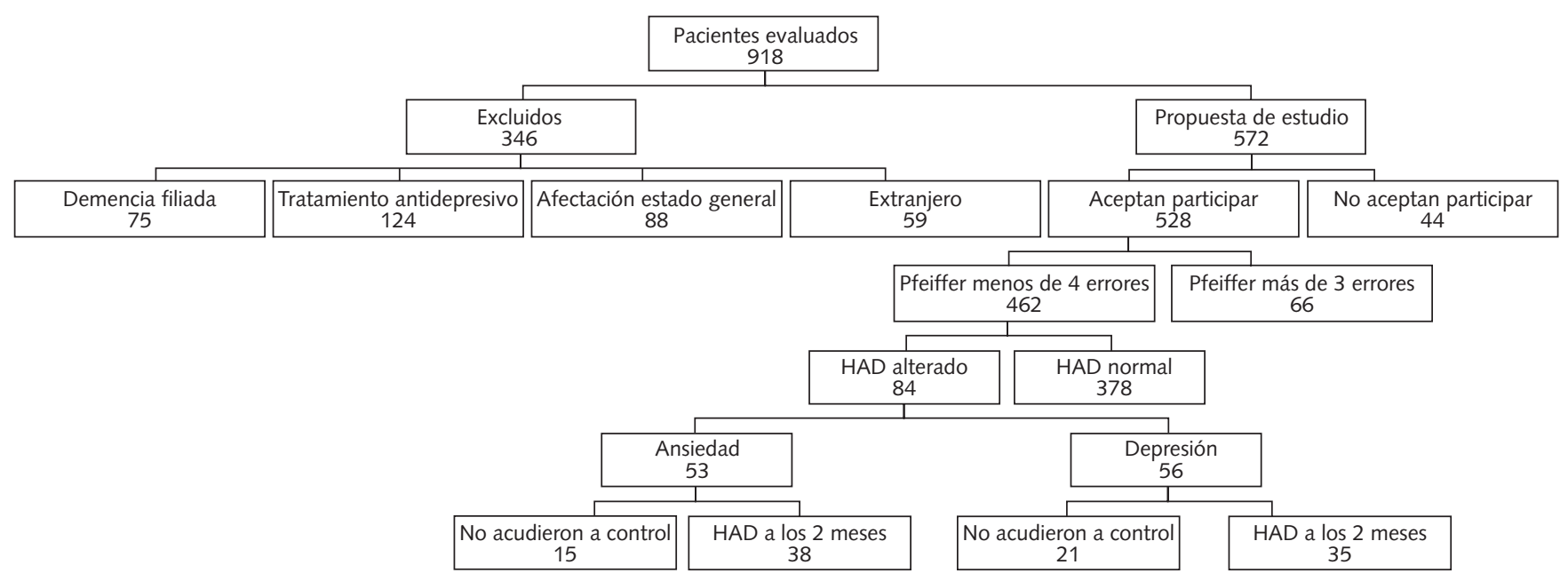

Fig. 1. Distribución de los pacientes evaluados en el estudio. 
TABLA I

CARACTERÍSTICAS DE LA MUESTRA ESTUDIADA Y DE LOS PARTICIPANTES QUE NO ACUDIERON AL CONTROL

\begin{tabular}{|c|c|c|c|}
\hline & $\begin{array}{l}\text { Si control a los } \\
2 \text { meses } n=57\end{array}$ & $\begin{array}{l}\text { No control a los } \\
2 \text { meses } n=27\end{array}$ & p \\
\hline Edad (1) & $65,7(16,4)$ & $67,4(17,9)$ & 0,66 \\
\hline Sexo femenino(2) & $24(42,1)$ & $11(40,7)$ & 0,90 \\
\hline Sin pareja (2) & $19(33,3)$ & $11(40,7)$ & 0,50 \\
\hline Con familia (2) & $45(78,9)$ & $24(88,9)$ & 0,26 \\
\hline Estudios primarios (2) & $49(86)$ & $21(77,8)$ & 0,34 \\
\hline Enfermedad crónica (2) & $49(86)$ & $21(77,8)$ & 0,34 \\
\hline Antecedente As (2) & $17(29,8)$ & $5(18,5)$ & 0,27 \\
\hline Antecedente Dp (2) & $14(24,6)$ & $5(18,5)$ & 0,53 \\
\hline Tto previo para As (2) & $10(17,5)$ & $3(11,1)$ & 0,44 \\
\hline Tto previo para Dp (2) & $7(12,3)$ & $4(14,8)$ & 0,74 \\
\hline Índice Charlson (1) & $2(1,9)$ & $2,8(2,5)$ & 0,15 \\
\hline Barthel (1) & $91(18,2)$ & $83,5(22,9)$ & 0,10 \\
\hline HAD-As Previo (1) & $7,8(4,7)$ & $5,1(4,2)$ & 0,015 \\
\hline HAD-Dp Previo (1) & $6,3(4,4)$ & $6,85(5)$ & 0,69 \\
\hline HAD-As Ingreso (1) & $9,2(4,1)$ & $8,5(4,7)$ & 0,47 \\
\hline HAD-Dp Ingreso (1) & $8(4,2)$ & $10,2(4,1)$ & 0,028 \\
\hline
\end{tabular}

As: ansiedad; Dp: depresión. (1) desviación típica, t de Student para muestras independientes. (2) (porcentaje medio) Chi cuadrado.

deterioro cognitivo mediante el test de Pfeiffer. En 84 participantes se obtuvo un HAD alterado, correspondiendo a 28 casos de As, 31 casos de Dp y 25 sujetos que puntuaron alto en As y Dp (Fig. 1).

De los 53 pacientes con As (aislada o con Dp) acudieron al control $38(71,7 \%), 22$ varones y 16 mujeres, con una edad media (desviación típica) de 62,2 $(15,4)$ años, y de los 56 pacientes con Dp (aislada o con As) se controlaron 35 pacientes (62.5\%), 22 varones y 13 mujeres, con una edad media (dt) de $68,1(15,5)$ años. Las características de la muestra estudiada se exponen en la tabla I, comparándolas con las características de los sujetos que no acudieron al control realizado a los dos meses. Los participantes controlados tuvieron puntuaciones más bajas en Dp durante el ingreso ( 8 frente a 10,$2 ; p=0,028)$ y más altas en As en las 3 semanas previas al ingreso (7,8 frente $5,1 ; \mathrm{p}=0,015)$, que los pacientes que no acudieron al control.

A los dos meses del alta 23 pacientes persistieron con síntomas de As (60,5\%; IC95\%: de 43,4 a 76). La persistencia de As se asoció con un nivel de estudios primarios y con la presencia de síntomas de As tres semanas antes del ingreso hospitalario, de modo que ninguno de los participantes sin As previa a la hospitalización se mantuvo con As a los dos meses del alta hospitalaria. Los antecedentes de un diagnóstico previo de As o Dp, o de un tratamiento para los mismos no significaron un mayor riesgo relativo (Tabla II). El valor de HADAs en las tres medidas efectuadas mostró una puntuación media (dt) de $10(4,2)$ tres semanas antes del ingreso, de 11,6 $(2,8)$ el cuarto día del ingreso, y de $9,1(5,2)$ puntos a los dos meses del alta (Fig. 2). La comparación de medias para datos pareados obtuvo diferencias estadísticamente significativas al enfrentar el HAD-As durante el ingreso con el previo ( $\mathrm{p}=$ $0,004)$ y con el posterior ( $p=0,002)$, pero no había diferencias entre el HAD-As previo y el posterior al ingreso $(\mathrm{p}=0,16)$.

Persistieron con síntomas de Dp a los dos meses 18 pacientes $(51,4 \%$; IC95\%: de 34 a 68,6). La presencia de sín-
TABLA II

VARIABLES ASOCIADAS A LA PERSISTENCIA DE SÍNTOMAS DE ANSIEDAD O DE DEPRESIÓN A LOS DOS MESES DE ALTA HOSPITALARIA

\begin{tabular}{|c|c|c|}
\hline Variables & $\begin{array}{c}\text { Riesgo Relativo (IC95\%) } \\
\text { Persiste } \\
\text { ansiedad } \\
\text { a los } 2 \text { meses }\end{array}$ & $\begin{array}{l}\text { Persiste } \\
\text { depresión } \\
\text { a los } 2 \text { mese }\end{array}$ \\
\hline Edad igual o mayor a 70 años & $1,17(0,49-2,83)$ & $2,05(1,01-4,14)^{*}$ \\
\hline Sexo femenino & $1,96(0,78-4,94)$ & $3,14(1,04-9,52)^{*}$ \\
\hline Estudios primarios o inferior & $1,50(1,05-2,15)^{*}$ & $1,31(1,01-1,70)^{*}$ \\
\hline No tener pareja & $1,47(0,55-3,92)$ & $0,79(0,30-2,11)$ \\
\hline Vivir con la familia & $0,85(0,62-1,17)$ & $0,94(0,68-1,32)$ \\
\hline Enfermedad crónica & $1,30(0,95-1,79)$ & $1,01(0,79-1,28)$ \\
\hline Indice de Charlson de 2 puntos o más & $1,20(0,56-2,54)$ & $1,73(0,83-3,64)$ \\
\hline Escala de Barthel menor a 100 & $2,17(0,71-6,62)$ & $3,46(1,16-10,31)^{*}$ \\
\hline Ansiedad asociada en el hospital & - & $1,21(0,58-2,53)$ \\
\hline Depresión asociada en el hospital & $1,44(0,62-3,30)$ & - \\
\hline Antecedente de ansiedad & $1,04(0,42-2,59)$ & $0,94(0,33-2,69)$ \\
\hline Antecedente de depresión & $2,94(0,73-11,75)$ & $0,76(0,24-2,35)$ \\
\hline No tratamiento previo por ansiedad & $0,75(0,55-1,01)$ & $1,02(0,71-1,46)$ \\
\hline No tratamiento previo por depresión & $0,70(0,48-1,03)$ & $1,08(0,82-1,42)$ \\
\hline Ansiedad alta en el hospital (HAD>10) & $1,85(0,95-3,60)$ & $1,13(0,42-3,03)$ \\
\hline Depresión alta en el hospital (HAD>10) & $1,63(0,36-7,35)$ & $1,42(0,64-3,13)$ \\
\hline HAD 3 semanas antes con ansiedad & $2,50(1,35-4,65)^{*}$ & $1,70(0,71-4,06)$ \\
\hline HAD 3 semanas antes con depresión & $2,94(0,73-11,75)$ & $2,36(1,20-4,64) *$ \\
\hline
\end{tabular}

*: riesgo relativo estadísticamente significativo.

tomas de Dp tres semanas antes del ingreso se asoció a mayor persistencia de la Dp. La edad (70 o más años), el sexo femenino, un nivel de estudios primarios y sobre todo un índice de Barthel durante el ingreso menor a 100, fueron otros factores asociados a la persistencia de Dp (Tabla II). El valor de HADDp en las tres medidas efectuadas mostró una media (dt) de 8 $(4,2)$ puntos tres semanas antes del ingreso, de 10,7 $(2,7)$ durante el ingreso, y de $8,4(5,2)$ a los dos meses del alta (Fig. $2)$. Se hallaron diferencias al enfrentar el HAD-Dp durante el ingreso con el previo $(p<0,001)$ y el posterior $(p=0,010)$, pero no era significativa la diferencia entre el HAD-Dp previo y el posterior al ingreso $(\mathrm{p}=0,58)$.

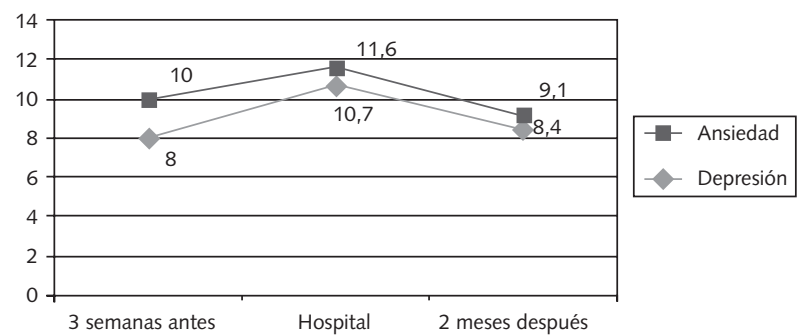

Fig. 2. Evolución de los valores de ansiedad y depresión mediante el cuestionario $H A D$, a las tres semanas antes del ingreso, durante el ingreso hospitalario y a los dos meses del alta. 


\section{DISCUSIÓN}

En nuestro estudio más de la mitad de los pacientes controlados con As durante la hospitalización, y la mitad de los pacientes controlados con Dp durante la hospitalización, permanecen sintomáticos dos meses después del alta hospitalaria. Estos resultados coinciden con investigaciones precedentes. Así, en valoraciones realizadas al mes del alta hospitalaria con muestras pequeñas (de 12 y 25 pacientes) se observó una persistencia de los síntomas de As y Dp en un 50\% de los casos $(23,24)$. En otra investigación, más de un $40 \%$ de los pacientes hospitalizados con síntomas de As o Dp permanecieron sintomáticos en el seguimiento al año del alta hospitalaria (25). Sin embargo, Creed et al (26) observaron que se mantenían los niveles de la escala HAD durante un seguimiento de 5 meses, concluyendo que estos trastornos son persistentes y no indicativos de distrés mientras la persona está hospitalizada.

En el presente estudio, cuando evaluamos la presencia de síntomas de As o Dp en tres momentos de la vida del paciente observamos un incremento de las puntuaciones medias del HAD en As y Dp durante la hospitalización, mientras que las puntuaciones medias de As y Dp de tres semanas antes del ingreso y de dos meses después del alta eran similares. En definitiva, apreciamos un incremento de lo síntomas de As y Dp durante el ingreso con tendencia a regresar al estado habitual tras el impacto de la hospitalización.

En la misma línea, observamos que una puntuación indicativa de As o Dp en la rememoración del HAD de tres semanas antes, se asoció a una persistencia de los síntomas de As o de Dp a los dos meses. Estos resultados se corresponden con la relativa estabilidad de los síntomas de As y Dp que se observa en la población general no hospitalaria donde se ha comprobado una correlación de la sintomatología ansiosa de 0,3 y correlaciones de la sintomatología depresiva de 0,5 y 0,67 , en seguimientos de hasta 10 años $(27,28)$.

La asociación de la Dp con los otros factores, como el sexo femenino, la mayor edad, la limitación para las activida- des de la vida diaria, y con un peor nivel cultural y económico ya es conocida y fue comentada en un estudio previo (8)

Las limitaciones de este estudio vienen condicionadas por el relativo bajo numero de casos estudiados y por las pérdidas del seguimiento, principalmente en los pacientes con mayor puntuación en Dp. Solo fueron valorados los enfermos que no recibían tratamiento, lo cual puede implicar que estudiamos un subgrupo de pacientes con As o Dp no detectados previamente por menor expresividad de los síntomas o de inicio reciente.

Identificar y tratar adecuadamente los procesos mentales de nuestros pacientes va a adquirir cada vez mayor importancia por su frecuencia, porque las estimaciones del "Global Burden of Disease Study" (29) sitúan a los trastornos mentales como la segunda causa de enfermedad e incapacidad en el mundo en el cercano año 2020. Y también por las consecuencias que conllevan, ya que el mantenimiento de la An y de la Dp puede facilitar un mayor deterioro funcional (30,31), y una peor calidad de vida relacionada con la salud (26,32-35).

Los pacientes con As tienen una percepción subjetiva de gravedad de la enfermedad superior a la de los pacientes sin As (8), a pesar de haberse demostrado una menor gravedad objetiva en los ansiosos $(8,16)$. En estudios longitudinales la sintomatología depresiva se ha asociado con mayor mortalidad cardiovascular y por todas las causas $(28,36-38)$. La curva de supervivencia en deprimidos y no deprimidos diverge a partir de los 4 meses (38), por lo que mantener una conducta observacional prolongada ante una Dp sería inadecuado.

Concluimos que la valoración del HAD previo al ingreso, con la ayuda de otros factores, como el estado funcional por la escala de Barthel, el sexo, la edad o el nivel de estudios, pueden ayudar a decidir la prescripción precoz de tratamiento o esperar a una nueva evaluación después del retorno del paciente a su hogar, cuando se detecta un caso de As o Dp durante una hospitalización. En cualquier caso, la persistencia de la sintomatología en más de la mitad de los pacientes obliga a su estrecho seguimiento tras el alta hospitalaria.

\section{Bibliografía}

1. Silverstone PH. Prevalence of psichiatric disorders in medical inpatients. J Nerv Ment Dis 1996; 184: 43-51.

2. Hansen MS, Fink P, Frydenberg M, Oxhoj ML, Søndergaard L, MunkJørgensen P. Mental disorders among internal medical inpatients prevalence, detection, and treatment status. J Psychosomatic Res 2001; 50: 199-204.

3. Clarke DM, Minas IH, Stuart GW. The prevalence of psychiatric morbidity in general hospital inpatients. Aust N Z Psychiatry 1991; 25: 322-9.

4. Lykouras E, Ioannidis C, Voulgari A, Jemos J, Tzonou A. Depresión among general hospital patients in Greece. Acta Psychiatr Scand 1989; 79: $148-52$.

5. Saravay SM, Steinberg MD, Weinschel B, Pollack S, Alovis N. Psychological comorbidity and length of stay in the general hospital. Am J Psychiatry $1991 ; 148: 324-9$.

6. Levenson JL, Hamer RM, Rossiter LF. Relation of psychopathology in general medical inpatients to use and cost of services. Am J Psychiatry 1990; 147: 1498-503.

7. Kunsebeck HW, Lempa W, Freyberger H. Identification of psychosomatic and psychic disorders in non-psychiatric in patients. Psychother
Psychosom 1984; 42; 187-94.

8. Grau Martín A, Suñer Soler R, Abulí Picart P, Comas Casanovas P Niveles de ansiedad y depresión en enfermos hospitalizados y su relación con la gravedad de la enfermedad. Med Clin (Barc) 2003; 120 : 370-5.

9. Fulop G. Anxiety disorders in the general hospital setting. Phychiatr Med 1990; 8: 187-95.

10. Haro JM, Palacín C, Vilagut G, Martínez M, Bernal M, Luque I, et al Prevalencia de los trastornos mentales y factores asociados: Resultados del estudio ESEMeD-España. Med Clín (Barc) 2006; 126: 445-51.

11. Beausang P, Syyed R. Screening for anxiety and depresión in adult general medical in-patients in a scottish district general hospital. Scot Med J 1998; 43; 177-80.

12. Koenig HG, George LK, Meador KG. Use of antidepressants by nonpsychiatrists in the treatment of medically ill hospitalised depressed elderly patients. Am J Psychiatry 1997; 154: 1369-75.

13. Kathol RG, Wenzel RP. Natural history of symptoms of depression and anxiety during inpatient treatment on general medicine wards. J Gen Intern Med 1992; 7: 287-93. 
14. Pfeiffer E. A short portable mental status questionaire for the assement of organic brain deficit in elderly patients. J Am Geriatr Soc 1975; 23: 433-41.

15. Martínez de la Iglesia J, Dueñas Herrero R,Onís vilches MC, Aguado Taberné C, Albert Colomer C, Luque Luque R. Adaptación y validación al castellano del cuestionario de Pfeiffer (SPMSQ) para detectar la existencia de deterioro cognitivo en personas mayores de 65 años. Med Clin (Barc) 2001; 117: 129-34.

16. Herrmann C. International experiences with the Hospital Anxiety and Depression Scale. A review of validation data and clinical results. J Psychosomatic Res 1997; 42: 17-41.

17. Zigmond AS, Snaith RP. The hospital anxiety and depression scale. Acta Psychiatr Scand 1983; 67: 361-70.

18. Bjelland I, Dahl AA, Haug TT, Neckelmann D. The validity of the Hospital Anxiety and Depression Scale. An updated literature review. J Psychosomatic Res 2001; 52: 69-77.

19. Tejero A, Guimerá EM, Farré JM, Peri JM. Uso clínico del HAD (Hospital Anxiety and Depresión Scale) en población psiquiátrica: un estudio de su sensibilidad, fiabilidad y validez. Rev Depto Psiquiatría Fac Med Barna 1986; 13: 233-8.

20. Quintana JM, Padierna A, Esteban C, Arostegui I, Bilbao A, Ruiz I. Evaluation of the psychometric characteristics of the Spanish Version of the Hospital anxiety and Depression Scale. Acta Psychiatr Scand 2003; 107: 216-21.

21. Charlson ME, Pompei P, Ales KL, MacKenzie CR. A new method of classifying prognostic comorbidity in longitudinal studies: Development and validation. J Chron Dis 1987; 40: 373-83.

22. Mahoney FD, Barthel DW. Functional evaluation: The Barthel Index Md State Med J 1965; 14: 61-3.

23. Pomerantz AS, de Nesnera A, West AN. Resolution of depressive symptoms in medical inpatients after discharge. Int J Psychiatry Med 1992; 22: 281-9.

24. Walker FB, Novack DH, Kaiser DL, Knight A, Oblinguer P. Anxiety and depression among medical and surgical patients nearing hospital discharge. J Gen Intern Med 1987; 2: 99-101.

25. Balestrieri M, Bisoffi G, De Francesco M, Eridani B, Martucci M, Tansella M. Six-month and 12 month mental health outcome of medical and surgical patients admitted to general hospital. Psycholog Med 2000; 30: 359-67.
26. Creed F, Morgan R, Fiddler M, Guthie E, House A. Depression and anxiety impair health-related quality of life associated with increased costs in general medical inpatients. Psychosomatics 2002; 43: 302-9.

27. Lovibond PF. Long-term stability of depression, anxiety, and stress syndromes. J Abnormal Psychology 1998; 3: 520-6.

28. Barefoot JC, Schroll M. Symptoms of depression, acute myocardial infarction, and total mortality in a community sample. Circulation 1996; 93: 1976-80.

29. Murray CJL, López AD. Evidence-based health policy-lessons from the Global Burden of Disease Study. Science 1996; 274: 740-3.

30. Covinsky KE, Fortinsky RH, Palmer RM, Kresevic DM, Landefeld CS Relation between symptoms of depression and health status outcomes in acutely ill hospitalised older persons. Ann Intern Med 1997; 126: 417-25.

31. Marcus SC, Olfson M, Pincus HA, Shear MK, Zarin DA. Self -reported anxiety, general medical conditions, and disability bed days. Am J Psychiatry $1997 ; 154: 1766-8$.

32. Lane D, Carroll D, Ring C, Beevers DG, Lip G. Effects of depression and anxiety on mortality and quality-of-life 4 months after myocardial infarction. J Psychosomatic Res 2000; 49: 229-38.

33. Booth BM, Blow FC, Loveland C, Cynthia A. Functional impairment and co-occurring psychiatric disorders in medically hospitalised men Arch Intern Med 1998; 158: 1551-9.

34. Loebach Wetherell J, Thorp SR, Patterson TL, Golshan S, Jeste DV, Gatz M. Quality of life in geriatric generalized anxiety disorder: a preliminary investigation. J Psychosomatic Res 2004; 38: 305-12.

35. Sherbourne CD, Wells KB, Meredith L, Jackson CA, Camp P. Comorbid anxiety disorder and the funcioning and well-being of chronically ill patients of general medical providers. Arch Gen Psychiat 1996; 53: 889-95.

36. Covinsky KE, Kahana E, Marshall HC, Palmer RM, Fortinsky RH, Landefeld CS. Depressive symptoms and 3-year mortality in older hospitalized medical patients. Ann Intern Med 1999; 130: 563-69.

37. Herrmann C, Brand-Driehorst S, Buss U, Rüger U. Effects of anxiety and depression on 5-year mortality in 5057 patients referred for exercise testing. J Psychosomatic Res 2000; 48: 455-62.

38. Hermann Lingen C, Klemme H, Meyer T. Depressed mood, physicianrated prognosis, and comorbidity as independent predictors of 1-year mortality in consecutive medical inpatients. J Psychosomatic Res 2001; 50: $295-301$ 